\title{
ILCEA
}

Revue de l'Institut des langues et cultures

d'Europe, Amérique, Afrique, Asie et Australie

42 | 2021

Le politiquement correct : tabous, normes, transgressions

\section{La formule politiquement incorrect dans le discours public : emplois, définitions discursives et enjeux socio-politiques et culturels}

The Politically Incorrect Formula in Public Discourses: Uses, Discursive

Definitions and Socio-Political and Cultural Issues

\section{Maria Saltykov}

\section{QpenEdition}

\section{Journals}

Édition électronique

URL : http://journals.openedition.org/ilcea/11573

DOI : 10.4000/ilcea. 11573

ISSN : 2101-0609

Éditeur

UGA Éditions/Université Grenoble Alpes

\section{Édition imprimée}

ISBN : 978-2-37747-251-2

ISSN : 1639-6073

Référence électronique

Maria Saltykov, «La formule politiquement incorrect dans le discours public : emplois, définitions discursives et enjeux socio-politiques et culturels », ILCEA [En ligne], 42 | 2021, mis en ligne le 31 janvier 2021, consulté le 01 février 2021. URL : http://journals.openedition.org/ilcea/11573; DOI https://doi.org/10.4000/ilcea.11573

Ce document a été généré automatiquement le 1 février 2021.

(c) ILCEA 


\title{
La formule politiquement incorrect dans le discours public : emplois, définitions discursives et enjeux socio-politiques et culturels
}

\author{
The Politically Incorrect Formula in Public Discourses: Uses, Discursive
}

Definitions and Socio-Political and Cultural Issues

Maria Saltykov

\section{Introduction}

1 L'expression politiquement correct n'est pas un néologisme. Fonctionnant depuis les années 1960 dans le discours américain, savant comme public, cette formule s'est propagée à partir des années 1990 et s'est étendue au discours européen (Toolan, 2003). Contrairement à politiquement correct qui a fait l'objet de divers travaux scientifiques, l'expression politiquement incorrect qui en dérive n'a pas été examinée en profondeur, et cela bien qu'elle apparaisse de plus en plus fréquemment dans l'espace public, dans différents domaines - politique, culturel, académique, etc. À cet égard, nous pourrions poser l'hypothèse qu'elle est une formule émergente dans le sens proposé par A. KriegPlanque (2009: 84), selon lequel la formule est une notion discursive caractérisée par sa forme figée, son caractère de référent social (elle devient un passage obligé sur un sujet donné) et son caractère polémique. La polémicité de l'expression figée politiquement incorrect se cristallise dans le discours public autour de questions socio-politiques, culturelles et éthiques, sans pour autant se réduire à ces domaines.

2 Le présent article se propose d'aborder plusieurs questions qui n'ont guère été traitées : quels sont les emplois de politiquement incorrect dans les discours savant, médiatique et numérique francophones et anglophones; quelles sont ses définitions en discours, à savoir celles « qui s'élabor[ent] dans le discours en situation » (Amossy, 2015 : 249 ; voir 
aussi Masasa, 2011); et surtout quels sont les enjeux socio-politiques de ces définitions?

3 Nous explorerons la formule politiquement incorrect sous plusieurs angles à la fois distincts et complémentaires. Premièrement, nous la définirons par le biais de la description de la formule de base opposée politiquement correct telle qu'on la trouve dans la littérature académique et les médias ; deuxièmement, nous aurons recours à une approche empirique qui consistera à décrire et à analyser les définitions discursives de la formule. Nous verrons ainsi comment elle "se voit assigner divers sens à travers son usage et cotexte [...] et en contexte " (Amossy 2015 : 249) et cela sur la base d'un double corpus : d'une part, celui de la littérature et des périodiques savants (le discours savant), d'autre part, celui rassemblé dans les médias et les données numériques contemporains (le discours médiatique, l'usage commun). Sur la base du corpus homogène sur lequel elle s'appuie, chacune de ces parties permettra une montée en généralité : elle nous aidera à dégager des tendances et des régularités propres (1) au métadiscours académique sur le $P C^{1}$ et à la notion de politiquement incorrect qu'on peut en induire; (2) à l'emploi de l'expression politiquement incorrect dans le discours savant ; (3) à l'usage commun de l'expression dans l'espace public (les médias).

Ces différentes perspectives présentent des points de convergence, grâce auxquels il sera possible de comprendre le sens global de la formule politiquement incorrect. Notre approche, qui vise une impartialité maximale sur un sujet très polémique, privilégiera sciemment la polyphonie des "voix » analysées. En d'autres termes, nous préférons «faire parler» les instances analysées plutôt que faire entendre une voix d'auteur engagé.

Dans le but d'esquisser une synthèse permettant de généraliser ce qui se dégage des interprétations du politiquement incorrect, nous privilégions une analyse qualitative effectuée à l'aide des instruments fournis par l'analyse du discours et de l'argumentation (Amossy, 2014 ; Charaudeau, 2005). Néanmoins, pour mettre en valeur la représentativité des résultats, nous avons exploré un corpus assez important, qui nous a permis d'introduire certaines données statistiques contribuant à expliquer les tendances d'usage de la formule. Cette étude entend ainsi offrir un panorama des sens qu'on attache à des fins argumentatives au politiquement incorrect dans les sociétés contemporaines, et entamer par là une réflexion sur son rôle et ses enjeux au sein du discours public. Elle se présente comme le prélude à une analyse des dynamiques discursives à l'œuvre dans les discours en situation qui font usage de la formule émergente, que nous n'aurons pas le loisir d'effectuer dans les limites de ce travail.

\section{Le politiquement incorrect en tant que réaction aux contraintes du politiquement correct}

6 Le politiquement incorrect est souvent perçu comme l'envers du discours politiquement correct, à savoir une transgression du « dogme » discursif et éthique. C'est maintenant, quand «l'histoire redevient houleuse» (Bock-Côté, 2019:16), que le politiquement incorrect émerge dans le discours public comme une formule indépendante, alors que le $P C$ s'est déjà lexicalisé et est devenu un lieu commun dans l'usage courant. C'est pourquoi nous analysons le traitement du $P C$ dans la sphère publique, et en particulier 
les critiques récurrentes qui lui sont adressées dans le cadre de toute une "offensive anti-pc » (Mangeot, $1997: 3$ ), pour en inférer les traits de l'incorrection.

Méthodologiquement, nous avons effectué à cette fin l'analyse d'un corpus constitué de travaux académiques qui traitent directement du politiquement correct, enrichi par une incursion dans les médias. Cet échantillon qui se veut pluridisciplinaire, contient les sources qui s'intéressent aux multiples manifestations du $P C$ : des livres (parmi d'autres: Battistella, 2005 ; Berman, 1993; Bock-Côté, 2019 ; Cameron, 2012 [1995]; Friedman \& Narveson, 1995 ; Guilleron, 2010 ; Lebouc, 2007 ; Wilson, 1995), des articles (Banning, 2014 ; Conway, Repke \& Houck, 2017 ; Duban, 1995; Fairclough, 2003 ; Gauthier, 1997 ; Halmari, 2011 ; Lopez Diaz, 2014 ; Mangeot, 1997 ; Miller, 1993 ; Strauts \& Blanton, 2015 ; Thom, 2010 ; Toolan, 2003; Western, 2016 et d'autres articles), des thèses de doctorat (Andary-Brophy, 2015 ; Dickson, 2017). On a analysé les sources qui datent de 1990-2019 pour accumuler et synthétiser un ensemble d'interprétations et de critiques récurrentes. Notre article présentera les grandes lignes de l'analyse et une partie des ouvrages essentiels ${ }^{2}$.

\subsection{La formule politiquement correct (PC)}

8 L'usage courant de la formule politiquement correct (politically correct) ou bien de sa version nominale la correction politique (political correctness), provient des mouvements sociaux émancipateurs (contre le racisme et le sexisme, les mouvements féministes et ceux pour les droits des minorités sexuelles) aux États-Unis à partir des années 1960. Initialement, le PC reflétait le désir de gérer l'emploi des termes susceptibles de blesser les sentiments des groupes considérés comme défavorisés. Dans un sens plus large, il s'agissait des tentatives pragmatiques de modifier les représentations sociales " déformées » et, conséquemment, le rôle social de ces groupes par le changement du champ lexical discriminatoire qui leur est associé (Cameron, 2012 [1995] : 142 ; Lopez Diaz, 2014). Vers les années 1990, l'épicentre du politiquement correct s'est graduellement formé au sein des universités américaines, allant de la propagation sur les campus des lignes directrices officielles prescrivant les formes d'adresse désormais considérées comme plus adaptées, à des débats acharnés sur la mise en question du «canon » occidental de l'enseignement supérieur traditionnel (Berman, 1993; Cameron, 2012 [1995] ; Duban, 1995 ; Friedman \& Narveson, 1995 ; Gauthier, 1997 ; Hamann, 1998 ; Miller, 1993).

9 Au cours des années 1980, le mouvement de la political correctness s'est propagé bien audelà des États-Unis pour s'installer en Europe (Toolan, 2003: 76), où cet « américanisme » a fait l'objet de vives critiques (Mangeot, 1997), en provoquant de multiples impulsions anti-PC. Ces dernières, en Europe ainsi qu'outre-Atlantique, ont donné naissance à l'idée de l'incorrection.

10 Ce qui réunit les sources qui abordent le $P C$ comme phénomène, c'est la constatation qu'il échappe à toute définition rigoureuse et universelle. Néanmoins, «quelques idéesclé, une série de clichés qui accompagnent en chapelet la mouvance $\mathrm{pc}$ » (Mangeot, 1997 :58), sont discernables à partir du corpus qui explicite le politiquement correct. Généralement, il s'agit de deux manifestations : verbale et comportementale (d'action), qui s'entrelacent au cours du procès discursif et argumentatif.

11 Le facteur premier qui mobilise le politiquement correct comme idéologie est l'évitement de la nomination catégorielle discriminante et en même temps, l'atténuation, jusqu'à y 
mettre un terme, de la position de domination (discernable dans le discours) qu'occupent des catégories reconnues comme privilégiées :

An extremely powerful set of norms [...], often called "political correctness" (or " $P C$ ") norms, explicitly attempts to remove negative group-relevant language. (Conway, Repke \& Houck, 2017 : 246)

La notion d'«affirmative action» - «discrimination positive» en français - est la projection pragmatique de ce principe sur les pratiques quotidiennes.

n autre impératif restrictif du politiquement correct est le contournement ou l'adoucissement de ce qui pourrait être perçu comme une transgression des principes ou des codes éthiques tacites propres à un type/genre communicationnel, des conventions de politesse, et surtout comme une nomination offensive, des propos choquants ou provocateurs, une violation des tabous et des sensibilités sociales, de la violence verbale (hate speech) (Conway, Repke \& Houck, 2017 ; Gauthier, 1997 ; Reinelt, 2011 ; Wilson, 1995). Nous pouvons regrouper ces démarches dans le cadre des normes culturelles de comportement dont la correction politique fait partie dans les communautés démocratiques (Conway, Repke \& Houck, 2017 : 245).

La sensibilité aux conventions culturelles de l'auditoire renvoie à une autre fonction du $P C$ qui consiste à développer la communication dans les sociétés par l'ouverture à la diversité, l'inclusion, la tolérance. Ces valeurs, fondées sur la valeur clé duPC - l'égalité, sont généralement associées dans le corpus savant aux forces politiques de l'aile gauche, au point que cette fusion devient le paradigme dominant (Fairclough, 2003 ; Strauts \& Blanton, 2015 ; Western, 2016 ; Wilson, 1995, entre autres). largement explorés: les euphémismes, perçus comme "the main tool of political correctness" (Halmari, 2011: 828); la terminologie et les codes spécifiques; toutes sortes de périphrases et de circonlocutions ; l'acronymie et les apocopes ; les chiffres ; des moyens syntaxiques, etc. (Cameron, [1995] 2012 ; Lebouc, 2007).

Les critiques dont les auteurs accablent le $P C$ et qui servent de prémisses au politiquement incorrect, sont nombreuses - elles manient l'accusation, la péjoration ou la dérision.

Dans le discours public, une partie des auteurs étudiés ont tendance à amalgamer le $P C$ et la censure. Dans ce sens, ils traitent le PC comme un assaut contre la liberté d'expression et de pensée, et comme la répression d'un débat public libre, l'imposition de bornes artificielles (Bock-Côté, 2019 ; Strauts \& Blanton, 2015 ; Western, 2016, etc.). Ainsi, Western considère que l'une des tâches du $P C$ est " to protect marginalised and oppressed groups against racism, homophobia, misogyny (at a cost of free speech and free thought) » (2016: 70).

Dans le cas où la political correctness apparaît sur le fond d'une idéologie politique, ses opposants évoquent souvent le radicalisme, principalement du camp politique "gauchiste». Ils dénoncent une "cultural and discursive intervention labelled as 'PC'» (Fairclough, 2003:25) qui consiste dans l'imposition de l'égalitarisme, de la pensée unique policée, de la propagande (Bock-Côté, 2019; Friedman \& Narveson, 1995 ; Wilson, 1995). Quant au langage $P C$ politique, les auteurs de ce «camp » le critiquent en raison de son assimilation à la "langue de bois" standardisée des politiciens : «[an] empty speech acts linked to a misplaced emphasis on language and not action » (Banning, 2014 : 195 ; et aussi Lopez Diaz, 2014, etc.). 
L'atténuation des différences dans le discours $P C$ conduit, selon ses critiques, à l'effacement des individualités, et, éventuellement, à entraver la communication au lieu de la favoriser (Conway, Repke \& Houck, 2017). De même, les auteurs sceptiques envers le $P C$ évoquent le double standard qui promulgue l'égalité des droits, mais ne prend en réalité en compte qu'un côté du conflit et débouche sur la discrimination inverse (Battistella, 2005 ; Wilson, 1995). Par ailleurs, cet argument anti-PC concerne la pratique de la "discrimination positive", selon lequel l'abus du PC dans les milieux professionnels comporte le risque de rabaisser les standards professionnels par des privilèges fondés sur le sexe ou l'origine, et non pas, par exemple, sur l'excellence académique. Cela peut «démoraliser ses bénéficiaires en mettant implicitement en doute leurs mérites" (Mangeot, 1997 : 59). Par ce même mécanisme, la victimisation des minorités ne produirait qu'une fixation de leur posture stéréotypée "basse » (Halmari, 2011 ; Western, 2016, parmi d'autres).

Enfin, une préoccupation essentielle liée au politiquement correct est son impact sur la langue ${ }^{3}$. De nombreux chercheurs dénoncent la déformation de la langue et du sens des mots (Lebouc, 2007 ; Mangeot, 1997 ; Thom, 2010 et d'autres). L'impact sur la langue est d'autant plus crucial pour le milieu français qu'il y a là un emprunt à l'anglais : par défaut « [1]'anglicisme atteste le péché de naissance » (Mangeot, 1997 : 57).

En résumant les caractéristiques et les critiques majeures du $P C$, nous pouvons déjà discerner des valeurs clés abstraites (Perelman, 2002 [1977]: 48) associées, par opposition, au politiquement incorrect et sur lesquelles se déploie le raisonnement politiquement incorrect $-\mathrm{PI}^{4}$.

\subsection{Le politiquement incorrect : les valeurs}

22 La valeur fondatrice du politiquement incorrect, revendiquée par les critiques du $P C$, est la liberté, contrairement à la valeur de l'égalité sous-tendant la correction. Effectivement, nous observons une dichotomie de type conflictuel : le culte de l'égalité exprimée par l'effacement des faits et des nominations susceptibles de heurter quelqu'un, contre la liberté interprétée comme le droit fondamental de s'exprimer et de penser la réalité dans toutes ses dimensions, même contradictoires, en rupture avec le conformisme d'une pensée unique. Une liberté politiquement incorrecte au niveau de la langue ne supporte aucune censure, aucun contrôle restrictif de l'extérieur. Les défenseurs du PI se revendiquent alors de la liberté d'expression émancipatrice, de la pensée critique, dispensées d'instance modératrice, quelle que soit la motivation de cette dernière.

Une autre valeur-phare réclamée par le politiquement incorrect est la vérité ou l'honnêteté dans le sens de la démonstration objective des faits, du "réel », fût-il désagréable ou inconvenant (Conway, Repke \& Houck, 2017). Dans le discours public, il s'agit de la revendication d'un ethos (dans le sens de Amossy (2010) : la construction de l'identité verbale dans le discours) sincère et ouvert exprimé par un franc-parler, par opposition au $P C$ associé à la " langue de bois ", connue pour dissimuler la pensée et travestir la réalité (Guilleron, 2010; Thom, 2010). Cette stratégie communicationnelle croît de façon prévisible en efficacité quand elle est présentée en opposition aux stratégies autant correctes que "mensongères" des instances concurrentes, en particulier dans la sphère politique. En revendiquant la posture incorrecte, on s'approprie également la vertu de l'audace ou la force d'exprimer et de proclamer des 
propos impopulaires - provocateurs ou même tabouisés (Conway, Repke \& Houck, 2017).

Si nous revenons à la dimension de l'action $P C$ dont l'une des expressions empiriques est «l'action affirmative», nous déduirons que pour ceux qui s'opposent au $P C$, ces pratiques ne sont rien d'autre que des manifestations d'un manque de professionnalisme qui balaie toute considération de mérite (Friedman \& Narveson, 1995). Ce serait donc une source d'injustice au sein des lieux professionnels. Ainsi, la position du politiquement incorrect dans la sphère professionnelle sera associée par ses promoteurs à une approche qui recherche l'objectivité dans l'évaluation du mérite et qui repose sur la pensée critique.

Il s'agit là de valeurs spécifiques affiliées au politiquement incorrect, basées sur l'intolérance envers le $P C$ jugé omniprésent dans l'espace public - donc, d'un niveau que nous pouvons qualifier d'idéologique. Mais la formule PI, comme son antipode, a de multiples dimensions qui ne cessent de se développer. Ainsi, au niveau verbal et par contraste avec le $P C$, le politiquement incorrect se manifeste par un discours qui transgresse les normes de la politesse, de l'éthique ou de la pertinence en vigueur dans une situation de communication.

Nous nous sommes en un premier temps appuyée sur les recherches consacrées au PC citées plus haut dans la présente partie, et en particulier sur sa critique, afin de dégager une vision synthétique des fondements du politiquement incorrect. La partie qui suit ajoutera une compréhension pratique de la formule qui fonctionne dans l'espace public, et de ses enjeux argumentatifs.

\section{L'approche empirique descriptive : la définition discursive du politiquement incorrect}

27 Faute de définitions lexicographiques et de théorisations cohérentes de l'ordre de celles qu'on trouve sur le $P C$, il nous faudra recourir ici à la définition discursive (Amossy, 2015 ; Masasa, 2011) de la formule politiquement incorrect afin de saisir son sens argumentatif. Sur la base d'un double corpus - celui rassemblé dans les médias écrits et numériques, mais aussi une série d'occurrences puisées dans la littérature savante on s'attachera à cerner une définition "naturelle» (Amossy, 2015:250) "qui s'élabor[e] dans le discours en situation" (ibid.: 249). Dans les circonstances d'énonciation concrètes, la formule «se voit assigner divers sens à travers son usage et cotexte [...] et en contexte»(ibid.) ainsi que (en suivant Masasa), à travers sa « temporalité » (ibid. : 19).

Notre approche comprend l'analyse des usages argumentatifs de la formule politiquement incorrect(e/s/es) (politically incorrect), de la locution nominale l'incorrection politique (political incorrectness) et de l'usage des dérivés tels que: non-politiquement correct(e/s/es), non-PC et autres variantes, - et la recherche des régularités d'emploi possibles et pertinentes. Logiquement, le critère formel de sélection des sources pour l'analyse a été l'inclusion de la formule recherchée dans ce corpus primaire. 


\subsection{Sources académiques et expertes} Le livre de Friedman et Narveson (1995) donne un panorama des avis sur l'impact du PC sur les campus universitaires; le livre de Cameron (2012 [1995]) représente le PC comme l'une des pratiques prescriptives utilisées dans le cadre de la «verbal hygiene ». La plupart des articles inclus dans ce corpus sont consacrés aux différentes manifestations du $P C$ (deux articles en français, huit en anglais; dont la plupart, six articles, datent des années 2011-2017, indiquant la croissance de son usage ; un de 2003 ; deux articles de 1997-1998 et un article témoignant des premiers usages en 1992).

31 Au niveau verbal, la locution adjectivale de la formule politiquement incorrect(e/s/es) (politically incorrect) prédomine dans les sources primaires dans les deux langues (près de 150 cas dans le corpus scientifique), tandis que la locution nominale apparait beaucoup moins ( 15 occurrences environ). Cela indique l'usage lexical principalement attributif de la formule : elle s'applique aux notions comme le langage, la culture, les attaques, les voix, et aussi politically incorrect speech, language, words, phrases; on trouve même un politically incorrect tribe (Western, 2016).

Les auteurs sont assez créatifs dans la production des formes périphrastiques et nuancées de la formule. Ainsi, nous remarquons que, dans le corpus présent, l'incorrection peut avoir des degrés. À côté des versions plus attendues, comme plus/ moins/presque politiquement incorrect, par exemple, Strauts et Blanton (2015) parlent de not at all/extremely politically incorrect. Les auteurs utilisent assez naturellement les formes périphrastiques dérivées, comme non-political correct(ness) (Friedmant \& Narveson, 1995 ; Toolan, 2003), ainsi qu'acronymiques : anti-pc (Mangeot, 1997), un-p.c. (Toolan, 2003), non-PC (Halmari, 2011) et même PIC (Western, 2016). La variété des degrés souligne le caractère subjectif du repérage de l'incorrection et de ses dimensions (lexique/action : «deeds (behaviour and policy) [and] words » [Toolan, 2003 : 70]).

L'usage de la formule politiquement incorrect dans les sources primaires scientifiques se caractérise par une neutralité relative du ton par rapport au corpus médiatique. Néanmoins, les auteurs critiquent aussi bien le PI que son envers.

La signification du politiquement incorrect est conditionnée par les circonstances communicationnelles de sa mise en fonctionnement. Nous observons ainsi avec le temps une évolution de ses domaines productifs. Pendant les années 1990, on évoquait le PI dans le contexte de l'imposition du PC dans le milieu universitaire (Boetcher Joeres \& Laslett, 1992 ; Friedman \& Narveson, 1995, Hamann, 1998, etc.) et à l'égard du discrédit de la lutte minoritaire, surtout contre le racisme et dans le cadre des mouvements féministes (Boetcher Joeres \& Laslett, 1992 ; Cameron [1995], 2012, Mangeot, 1997, etc.). L'usage de la formule pendant cette période-là est épisodique (20 cas environ), souvent entre guillemets, comme il convient à une unité lexicale nouvelle et peu commune. 

comme une révolte politique, mais aussi culturelle contre des normes de communication restrictives (Conway, Repke \& Houck, 2017), mobilisée par l'esprit de contradiction ; comme le courage de prendre des risques (Western, 2016: 73); comme l'audace d'aller à contre-courant et d'énoncer des choses impopulaires (Florence, 2015). On peut rapporter cette revendication à un ethos du type "combattant contre le système », le contenu du « système » étant spécifié selon les enjeux.

41 Cependant, le prix de cette combativité présentée comme quasi héroïque par les promoteurs duPI est parfois trop élevé aux yeux de la partie de la société principalement sensible aux valeurs humanistes et démocratiques, ainsi qu'aux normes éthiques gérant la communication. Dans le corpus primaire académique relativement récent, le discours PI reçoit de nombreuses critiques disqualifiantes, susceptibles de problématiser son déploiement argumentatif et de nuire à son effet persuasif. 

nuisible pour la société dans la mesure où il alimente les stéréotypes, comme le racisme et le sexisme (Strauts \& Blanton, 2015) et renforce les discriminations envers d'autres groupes: classes défavorisées, groupes régionaux, personnes avec des défauts physiques, etc. (ibid.). Il faut marquer la disposition des publications plus récentes à accentuer l'aspect verbal de l'(in)correction. Pour certains, elle est le fait du choix des mots (Florence, 2015) et même des constructions syntaxiques (Halmari, 2011).

Le corpus de la présente partie dévoile aussi certaines spécificités de perception culturelle. Alors que, pour l'Amérique du Nord, le $P C$ a toujours concerné la lutte pour les droits des minorités avec ses crises et rebondissements, le public français y a vu une idéologie profondément étrangère (Boetcher Joeres \& Laslett, 1992 ; Mangeot, 1997 ; Toolan, 2003, entre autres). Si, aux États-Unis, le mouvement PI a résulté de la critique des aspects tenus pour des défauts inhérents à l'idéologie de la political correctness, en France, le produit américain a été condamné par défaut: «le "politiquement correct" n'a jamais eu très bonne presse » (Mangeot, 1997 : 57). Ainsi, outre la critique standard, le discours anti- $P C$ français comporte aussi cette nuance de coercition, de l'imposition d'un élément étranger à une culture qui se veut fondée sur l'esprit libre et la pensée critique.

\subsection{Corpus médiatiques et numériques}

Le corpus médiatique comprend les articles marqués par les occurrences de la formule politiquement incorrect publiés dans dix journaux renommés qui ont de l'impact sur l'opinion publique dans les pays où ils sont édités et au-delà (nous marquerons de même les périodes disponibles techniquement pour la recherche) : Le Nouvel Observateur (2000-2019), Libération (2013-2019), Le Monde (1992-2019), Le Figaro (2003-2019), Le Point (2014-2019), Marianne (2007-2019) (France); The New York Times (1988-2019), The Washington Post (1992-2019), Newsday (2015-2019) (États-Unis); The Telegraph (2004-2019) (Royaume-Uni). Les orientations idéologiques et les styles de présentation diffèrent dans cet échantillon ${ }^{6}$, ce qui est censé assurer l'objectivité nécessaire à une généralisation sur l'emploi de la formule recherchée. En ce qui concerne les corpus numériques, nous avons eu recours aux commentaires contenant la formule politiquement incorrect sur les réseaux sociaux, blogs et forums.

45 La période sur laquelle nous avons pu examiner chaque journal dépend de la disponibilité des archives. En général, nous nous sommes penchée sur les tendances des dix dernières années (dès le début des années 2010) - période où un essor saillant de la formule a eu lieu - en parcourant néanmoins la décennie précédente et les occurrences notables plus anciennes (années 1990). La quantité d'articles varie d'une source à l'autre de 50-60 à presque 300 items par journal' .

La vision médiatique (et donc publique) de l'essence même du politiquement incorrect est, dans l'ensemble du corpus, mixte dans le sens où elle intègre les deux interprétations déjà évoquées dans le corpus primaire scientifique: la dimension verbale (le style discursif ou les paroles concrètes) et la dimension comportementale ou d'action. Dans les deux cas, la formule fonctionne librement, faisant partie d'énoncés et de métaphores diversifiées, en tant que caractéristique personnelle (par exemple, l'ethos d'un homme politique) ou trait distinctif d'un groupe (racisme, sexisme, etc.), en tant que phénomène concret (une réforme) ou abstrait (des idées).

ILCEA, 42 | 2021 
Contrairement à l'emploi du politiquement incorrect au sein du discours savant, où il a été principalement considéré dans le contexte du $P C$, les médias et les corpus numériques en font un usage indépendant, manifestant ainsi un stade essentiel de sa lexicalisation. Cela est aussi perceptible au niveau graphique: durant les années 2000, l'usage des guillemets et des italiques pour marquer l'étrangeté de la formule a diminué. Au niveau lexical, le nombre impressionnant des dérivés qui font allusion au politiquement incorrect, témoigne de son ancrage ferme dans la perception publique. En voici quelques exemples: économiquement, socialement, sexuellement, religieusement, éthiquement, historiquement, affectivement, scientifiquement, judiciairement, racialement, poétiquement, moralement, idéologiquement, artistiquement incorrect, anti-politiquement correct, anti-PC, politiquement inacceptable, et même gauchement, homosexuellement, policièrement incorrect ${ }^{8}$; et en anglais : romantically, sentimentally incorrect, PI, un-pc, et d'autres.

De même, comme dans le cas du corpus savant, les médias déploient toute une échelle de degrés du PI qui varie de: un peu, pour le moins, presque, encore plus politiquement incorrect - et jusqu'à politiquement très, le plus, plus que, vraiment, carrément, totalement, parfaitement, définitivement, férocement, radicalement, beaucoup trop, tout à fait incorrect ; the most, totally, too politically incorrect. On trouve encore : le PI [qui] atteint des sommets, ôcombien politiquement incorrect, politiquement incorrect en diable; super-over-mégaincorrection, etc. Une telle variabilité prouve que la formule est profondément enracinée dans la doxa commune.

Le politiquement incorrect en tant que formule reçoit dans l'usage des médias des interprétations contradictoires, qui vont de l'admiration au dégoût. Cela dépend moins de l'orientation idéologique de la revue, que de la rubrique où l'on use de la formule (sans, pour autant, niveler les prédispositions idéologiques plus ou moins importantes des revues à l'(in)correction). Deux rubriques se partagent la primauté : la culture et la politique (ce qui se dégage également du corpus savant), complétées par les sujets de société. Notre article présentera l'analyse détaillée de l'emploi de la formule dans la sphère politique. Il convient de mentionner d'emblée quelques statistiques générales pour donner une idée de la situation dans son ensemble.

Nous avons fixé des pics maximums dans la chronologie de l'usage général de la formule. Dans notre corpus, la première hausse s'observe dans les années 2006-2009 avec un pic en 2008. Ensuite, on constate une progression à partir de 2012, une hausse vers 2015 et un sommet important en 2016. Légèrement en baisse en 2017 (tout en restant à un niveau élevé), l'usage a atteint un nouveau sommet en 2018, comparable à celui de 2016. La recherche inclut des données jusqu'en octobre 2019 et nous pouvons déjà constater la tendance à un niveau d'usage assez élevé9.

Dans le contexte politique, nous avons observé un conditionnement contextuel et temporel de la formule politiquement incorrect par des événements précis. Ses pics d'usage ont été provoqués par des événements qui ont ému la société : la Présidentielle française de 2007, la campagne et la présidence de Nicolas Sarkozy (2006-2009); les attaques terroristes (2015) ; la Présidentielle américaine (2015-2016) et la présidence de Donald Trump ; les élections de mi-mandat aux États-Unis en 2018. Les autres sujets qui ont attiré des commentaires contenant la formule sont les suivants: la crise économique mondiale (2008-2009) ; l'outrage au drapeau français sur une photo primée lors d'un concours de la FNAC (2010) ; le Front National et la figure de J.-M. Le Pen; le président Obama ; les fusillades aux États-Unis ; le port de la burqa et du burquini ; la 
campagne \#metoo; Boris Johnson en tant que Premier ministre du Royaume-Uni; le manifeste de Greta Thunberg devant l'ONU en septembre 2019.

Les médias français accordent en moyenne $57 \%$ des mentions aux publications consacrées à la politique en France (environ 180 cas), 20 \% exclusivement à la politique nord-américaine (environ 60 cas) et $23 \%$ aux événements mondiaux (environ 70 cas). Ces statistiques indiquent encore une fois que les Français maintiennent une association forte entre les États-Unis et les notions «importées». Aux États-Unis, les journaux se concentrent plutôt sur la politique domestique ( $88 \%$ ) (environ 115 cas) et n'accordent que $12 \%$ (environ 17 cas) aux sujets internationaux.

Dans le cadre du contexte politique, les médias de notre échantillon caractérisent par le politiquement incorrect non seulement des personnes, images, personnages, présidents, candidats, hérauts et adeptes de l'incorrection, mais aussi les manifestations qui dérivent de leur activité politique: réformes, débats, discours, déclarations, positions, phrases, répliques, ton politiquement incorrects :

Dernière provocation du très politiquement incorrect candidat à l'investiture républicaine : se moquer d'un handicapé. La blague de trop ? (Le Point, 26 novembre 2015)

Pour cet ex-UMP [Sébastien Chenu], l'électorat frontiste attend des débats «non aseptisés » et " politiquement incorrect ». (Le Figaro, $1^{\text {er }}$ août 2017)

En anglais, on parlera de politically incorrect reforms, statements, language, approach, topics, remarks, events, speech, rhetoric, style, outbursts, leadership :

Another Reason editor, Nick Gillespie, contrasted the libertarian spirit of "cosmopolitanism and tolerance" with Trump's demonization of undesirables-and with the left's anti-pluralist drive to silence politically incorrect speech. (Newsday, 23 février 2017)

L'attitude médiatique envers l'ethos ou les démarches PI a remarquablement évolué avec le temps, et, encore une fois, avec l'avènement de Donald Trump qualifié de «nouveau héraut du politiquement incorrect » (Le Monde, 13 mars 2018). "Defender of political incorrectness» (Washington Post, 2016), il est devenu une figure symbolique. " $[\mathrm{He}]$ has made a brand of being politically incorrect " (Washington Post, 2018) au point que son discours spécifique est devenu un lieu commun : les articles emploient les locutions comme "Trump-type ", "Trump-like» et même "the Trumpiest » types du discours politique (Washington Post, 2017, parmi d'autres).

Pourtant, il n'y a pas si longtemps, le politiquement incorrect dans le discours ou dans le positionnement politique avait été associé par les médias du corpus avec un risque sérieux de nuire à la réputation du sujet. Ils impliquaient pour cette raison que les acteurs politiques devaient avoir peur de paraître incorrects. Par exemple, la figure conforme de Hillary Clinton pendant sa campagne présidentielle de 2016 a donné l'impression qu'elle craignait d'être PI (Newsday, 2016). Voici d'autres cas :

Dans la bouche de Godfrey Bloom, Bongo-Bongo Land [les pays pauvres], cela semblait être quelque chose proche de l'Afrique vue par Tintin au Congo, une région arriérée, peuplée de sauvages. Depuis, l'ancien élu a réussi l'exploit peu glorieux d'être tellement politiquement incorrect qu'il s'est fait mettre à la porte du UKIP, et n'est plus député européen. (Le Monde, 4 avril 2015)

Scheer has thus spent the first few months of his low-profile leadership wishing immigrant communities a happy this-or-that, and trying to distance himself from the politically incorrect outbursts of some of the less-disciplined members of his caucus. (The Washington Post, 13 octobre 2017) 
Pour quelle raison les démarches politiquement incorrectes étaient-elles considérées par les médias comme risquées? Ils associaient le côté idéologique du PI au racisme, sexisme, antisémitisme, chauvinisme, et à l'homophobie, la xénophobie, la misogynie, l'islamophobie, la négrophobie, etc. - pratiques socialement condamnables, fondées sur les préjugés, les clichés et les stéréotypes discriminatoires :

D'Éric Zemmour à Marine Le Pen, de Claude Guéant à Robert Ménard, le fond de l'air est à un «politiquement incorrect » raciste et conservateur. (Le Monde, 29 avril 2011)

Dans le cas de la gangrène islamophobe comme de celui du poison antisémite, l'installation publique de manichéismes concurrents a bénéficié du développement d'une compétition des antiracismes, entre lutte contre l'antisémitisme et combat contre l'islamophobie. Et chez certains intellectuels, pris par les passions tristes du " politiquement incorrect ", une morale de l'irresponsabilité participe à mettre de l'huile dans les mécanismes de ces dérèglements idéologiques. (Libération, 10 novembre 2017)

Le politiquement incorrect devient ainsi une sorte de justification de la violence verbale.

Sur le plan de l'idéologie politique, le discours PI est toujours conçu comme l'attribut des forces (néo)conservatrices de la droite, les républicains, qui s'opposent discursivement à un «liberal dogma ». Ces dernières années, le politiquement incorrect est aussi présenté dans les médias comme l'un des symptômes du populisme.

qui concerne les caractéristiques de l'acteur politique politiquement incorrect, cette tendance est assimilée dans les médias à l'insolence et l'incivilité, à un style d'expression intolérant, dérangeant, cynique et même haineux, qui n'hésite pas à heurter les sensibilités sociales. Les médias anglophones le caractérisent par : rudeness, impoliteness ; brash, blunt, boorish style; verbal cruelty and humiliation.

Ces caractéristiques peu flatteuses sont néanmoins présentées comme une construction d'image consciente, ce qui se confirme par le mot "provocation» et ses dérivés, attachés aux manifestations du PI. La presse évoque la "willingness» des hommes politiques à se présenter en politiquement incorrects: nous observons des formulations comme "self-proclaimed ", "self-described ", "brand himself as ", " relish being " politically incorrect, qui rendent compte de la «fierté » attachée à cette attribution risquée (nous avons mentionné plus haut une co-utilisation récurrente du mot «risque » avec ses dérivés dans les locutions avec la formule en question). À l'aide de la rétorsion rhétorique, les politiciens parviennent (et les médias le constatent) à convertir cet ethos politique jugé risqué et impopulaire (Le Figaro, 2006) au premier degré, en un acte d'audace et de courage (ils se permettent d'« oser » le PI, voir Le Monde, 2007-2008 et d'autres). Dans le même temps, ils s'approprient les valeurs fondatrices du PI, telles que la liberté de pensée, la sincérité, la force de prononcer des « vérités » impopulaires.

Dans les termes du discours journalistique sur le PI, celui qui ose briser les tabous et jouer avec les codes se voit affecter la mission de révéler au grand public une " réalité » politiquement incorrecte, la "ugly reality » et d'obtenir de la part des médias le titre de «brutally honest » «truth teller ». Il adopte une stratégie contrastive et se positionne en «outsider » de l'establishment politique et même en «iconoclaste » du système. Ainsi, dans ce système discursif, il propose symboliquement à l'auditoire une nouvelle vision $\mathrm{du}$ monde et $\mathrm{du}$ fonctionnement politique: franche, réaliste même dans toute sa laideur, dépourvue de conventions imposées d'en haut. 
63 À cette fin, il s'appuie rhétoriquement sur la liberté de pensée et d'expression qu'il choisit d'opposer à la pensée policée. Cette émancipation hors du conformisme éthique doit lui conférer une « authenticité » sur le champ politique. Une partie importante des internautes partage cet avis, par exemple :

Que c'est bon le politiquement incorrect [...]. Trump ou pas, enfin quelqu'un qui ose dire ce qu'il pense. [...] J'approuve, juste pour la liberté de penser... (Forum du Figaro, 2016)

D'après les commentaires critiques, c'est avec cet ensemble de qualités que les « chantres » du politiquement incorrect en politique réussissent à « séduire » le public en instrumentalisant « leurs frustrations et inquiétudes» (Nouvel Observateur, 2016-2017). En 2015, Newsday affirmait que "Americans want candid, brutally honest, sometimes politically incorrect leadership ». En outre, la capacité de défier le système, renforcée par un langage fort et combatif, implique aussi la qualité de "strong leadership", paraphrasée par les médias comme la capacité de prendre nettement position - «take a stand ", " having a voice ", "standing up for oneself », « [being] strong and fearsome », etc.

Cependant, ce côté fort attrayant médiatiquement de l'ethos PI conduit à ce que le discours auparavant transgressif devient aujourd'hui un nouveau discours dominant. "La bien-pensance a changé de camp, la pensée réactionnaire est à la mode », dit Léa Salamé au journal Le Monde (2014). La tâche de combattre le système se systématise à son tour. Le Figaro dénonce ainsi en 2015 une "pseudo-authenticité » du candidat Trump. De même, une partie critique des internautes s'indignent du fait que le politiquement incorrect est en train de devenir un nouveau mainstream public :

La liberté de pensée est-elle sous la menace d'un contre-pouvoir sournois, qu'il

faille se montrer inflexible devant le politiquement incorrect? (Forum du Figaro, $2009[$ sic $]$ ).

On trouve beaucoup d'autres témoignages de ce type.

Mais les adeptes du PI restent aussi motivés, à côté des autres enjeux, par la garantie d'une forte présence médiatique : "Les champions du "politiquement incorrect" sont les rois du prime time. [...] Leur posture, qui se prétend rebelle, jouit d'une domination sans partage. » (Le Monde, 2013) Cela vaut non seulement pour les hommes et femmes politiques, mais aussi pour la sphère de la culture, que nous n'analyserons pas ici faute de place.

\section{Conclusion : Enjeux du politiquement incorrect dans le discours public contemporain}

Le présent article a permis d'exposer la fonction argumentative de la formule émergente politiquement incorrect à partir de son fonctionnement dans les discours publics contemporains des pays francophones et anglophones. Nous avons tenté de saisir son rôle et ses enjeux, en montrant d'abord la façon dont le politiquement incorrect est né d'une réaction au politiquement correct, et en comparant ensuite plusieurs corpus qui éclairent les sens de la formule. En tant que formule, le politiquement incorrect reçoit dans la sphère publique des traitements divergents et même polémiques, et son rôle de référent social se fortifie, particulièrement dans la sphère de la politique. En considérant la formule sous plusieurs angles, nous sommes parvenue à dégager des régularités dans la façon dont le phénomène est perçu au sein des espaces proposés. 
discours public politiquement incorrect, et l'image de soi (ethos) qu'il construit du locuteur sur la base de valeurs spécifiques, visent à distinguer son porteur et à le différencier d'autres acteurs de l'espace public. Le politiquement incorrect non seulement prône une transgression des limites habituellement respectées, mais encore il propose une vision du monde alternative invitant à repenser ce qui est bon et pertinent, et ce qui ne l'est pas. C'est en juxtaposant les deux mondes - celui dit de la convention rigide et mensongère et celui dit de la liberté de l'esprit critique non biaisé, qu'on parvient à activer la force argumentative du politiquement incorrect. L'irrévérence se métamorphose par ce biais en authenticité, la polarisation sociale en l'exigence objective de notre temps.

Nous avons montré que l'argumentation PI doit se comprendre sur l'axe temporel. Ce n'est pas une stratégie stable et pérenne : une alternative n'est demandée que dans des conditions de changements profonds tant géopolitiques et idéologiques que culturels. Cet avis est abondamment documenté dans nos sources, expertes comme médiatiques, qui traitent de la formule, ainsi que dans celles qui analysent son antipode le PC. Nous nous permettrons à cet égard de reprendre la déclaration de N. Demorand, l'ancien patron de Libération, citée dans Le Monde (16 mars 2014) ; elle concerne initialement la télévision, mais peut avoir des applications plus générales: "Nous assistons à ce moment à la grande inversion : le politiquement incorrect est devenu correct. » Cette remarque appelle à prolonger l'étude de la formule, ainsi que de ses sphères d'influence.

\section{Références primaires}

\section{Corpus savant}

Sont répertoriées comme primaires les sources dans lesquelles l'expression politiquement incorrect ou ses dérivés est employée.

BOETCHER JOERES Ruth-Ellen \& LASLETT Barbara (1992), « On Being Labeled Politically (In)Correct: Introduction », Signs, 17(4), 779-780.

CAMERON Deborah (2012 [1995]), Verbal Hygiene, Abingdon : Routledge.

CONWAY Lucian Gideon, REPKE Meredith \& HoucK Shannon C. (2017), « Donald Trump as a Cultural Revolt Against Perceived Communication Restriction: Priming Political Correctness Norms Causes More Trump Support », Journal of Social and Political Psychology, 5(1), 244-259.

FloRence Joshua (2015), « A Phrase in Flux: The History of Political Correctness », Harward Political Review, en ligne sur <harvardpolitics.com/united-states/phrase-flux-history-politicalcorrectness/> (12 décembre 2020). 
FRIEDMAN Marilyn \& NARVESON Jan (1995), Political Correctness: For and Against, Lanham, USA : Rowman \& Littlefield.

HALMARI Helena (2011), « Political Correctness, Euphemism, and Language Change: The Case of 'People First' ", Journal of Pragmatics: An Interdisciplinary Journal of Language Studies, 43(3), 828-840.

HAMANN Jean (1998), « Politiquement incorrect? », Le Fil, Le journal de la communauté universitaire, en ligne sur <https://archives.nouvelles.ulaval.ca/Au.fil.des.evenements/1998/06.11/ politique.html> (12 décembre 2020).

LEBOUC George (2007), Parlez-vous le politiquement correct ?, Bruxelles : Éditions Racine.

MANGEOT Philippe (1997), « Bonnes conduites ? Petite histoire du " politiquement correct », Vacarme, 1(1), 57-59.

ReInelt Janelle (2011), « The Performance of Political Correctness », Theatre Research International, $36(2), 134-147$.

STRAUTS Erin \& BLANTON Hart (2015), « That's Not Funny: Instrument Validation of the Concern for Political Correctness Scale », Elsevier, Personality and Individual Differences, 80, 32-40.

ToolAn Michael (2003), « Le politiquement correct dans le monde français », Discourse \& Society, 14(1), 69-86.

WESTERN Simon (2016), « Political Correctness and Political Incorrectness: A Psychoanalytic Study of New Authoritarians », Organisational \& Social Dynamics, 16(1), 68-84.

\section{Corpus médiatique et numérique}

LE NOUVEL OBSERVATEUR, archives numériques (2000-2019), en ligne sur <www.nouvelobs.com>.

LiBÉRATION, archives numériques (2013-2019), en ligne sur <www.liberation.fr>.

LE MONDE, archives numériques (1992-2019), en ligne sur <www.lemonde.fr>.

LE FIGARO, archives numériques (2003-2019), en ligne sur <www.lefigaro.fr>.

LE PoINT, archives numériques (2014-2019), en ligne sur <www.lepoint.fr>.

MARIANNE, archives numériques (2007-2019), en ligne sur <www.marianne.net>.

THE NEW YORK TIMES, archives numériques (1988-2019), en ligne sur <www.nytimes.com>.

THE WASHINGTON POST, archives numériques (1992-2019), en ligne sur <www.washingtonpost.com>. NEWSDAY, archives numériques (2015-2019), en ligne sur <www.newsday.com>.

THE TELEGRAPH, archives numériques (2004-2019), en ligne sur <www.telegraph.co.uk>.

\section{Références secondaires}

Amossy Ruth (2010), La présentation de soi. Ethos et identité verbale, Paris : Presses universitaires de France.

Amossy Ruth (2014 [2000]), L'Argumentation dans le Discours, Paris : Armand Colin.

Amossy Ruth (2015), « La polémicité des définitions en discours. La formule "délégitimation

d'Israël” dans la presse française », G. Angermuller \& G. Philippe (dir.), Analyse du discours et 
dispositifs d'énonciation. Autour des travaux de Dominique Maingeneau, Limoges : Lambert Lucas Éditions, 249-259.

ANDARY-BROPHY Christine Anne (2015), Political Correctness: Social-Fiscal Liberalism and Left-Wing Authoritarianism (thèse de doctorat), University of Toronto, ProQuest Dissertations Publishing. Battistella Edwin L. (2005), Bad Language. Are Some Words Better Than Others?, New York: Oxford University Press.

Berman Paul (dir.) (1993), Debating P.C.: The Controversy over Political Correctness On College Campuses, New York : Laurel Trade Paperback.

BocK-CôTÉ Mathieu (2019), L'empire du politiquement correct, Paris : Les Éditions du Cerf.

ChARAUdeAu Patrick (2005), Le discours politique. Les masques du pouvoir, Paris : Librairie Vuibert.

Duban Jeffrey M. (1995), « Neurotic Political Correctness at Columbia University », The Journal of Blacks in Higher Education, 8, 78.

FAIRCLOUGH Norman (2003), « 'Political correctness': The Politics of Culture and Language », Discourse \& Society, 14(1), 17-28.

GAUTHIER Janel G. (1997), « La rectitude politique dans le milieu universitaire : plusieurs facettes, significations et conséquences », Canadian Psychology, 38(4), 202-203.

GUILLERON Gilles (2010), Langue de bois : décryptage irrévérencieux du politiquement correct et des dessous de la langue, Paris : Éditions Générales First.

KRIEG-PlANQUe Alice (2009), La notion de « formule » en analyse du discours. Cadre théorique et méthodologique, Besançon : Presses universitaires de Franche-Comté.

LOPEZ DiAz Monserrat (2014), « L'euphémisme, la langue de bois et le politiquement correct : changements linguistiques et stratégies énonciatives ", L'Information grammaticale, 143, 46-55.

MASASA Karina (2011), Contribution à la rhétorique des « formules magiques » dans les débats publics actuels sur la mondialisation : le cas de la polémique autour des concepts de " pauvreté » et de «solidarité » (thèse de doctorat), Université Bar-Ilan, Ramat-Gan.

MILLER Abraham H. (1993), « Political Correctness and American Higher Education », Politics, 13(1), 22-28.

PERELMAN Chaïm (2002 [1977]), L'empire rhétorique. Rhétorique et argumentation, Paris : J. Vrin.

Tном Françoise (2010), « Langue de bois et aphasie moderne », Hermès, 58(3), 129-133.

WILson John K. (1995), The Myth of Political Correctness, Durham : Duke University Press.

\section{NOTES}

1. Abréviation usuelle du politiquement correct.

2. L'analyse détaillée a été entreprise dans le cadre de la thèse de doctorat de Maria Saltykov sous la direction du Pr. Ruth Amossy. De même, la bibliographie complète de la présente partie, qui compte à peu près 100 sources, a été utilisée pour la thèse et ne peut être présentée que partiellement.

3. Ici, comme dans tout l'article, on tient compte de deux environnements linguistiques - francophone et anglophone. 
4. Ici et dans la suite, on se permettra, à l'exemple du $P C$, de raccourcir la locution politiquement incorrect à PI.

5. La publication n'est pas strictement académique, mais a une valeur intellectuelle et experte qui l'inscrit dans le bloc du présent corpus.

6. L'interprétation du PI en fonction des idéologies divergentes des journaux serait un sujet intéressant pour une étude séparée en vue de développer les thématiques abordées dans le présent article.

7. Étant donné le volume du corpus, et les restrictions formelles de cet article, nous avons dû renoncer à citer dans le texte les titres d'articles et leurs auteurs, sachant qu'on peut les compter par dizaine(s) pour chaque tendance.

8. Ces derniers adverbes, malgré leur forme inusitée en français, font partie des formulations nuancées contextuelles de divers auteurs.

9. En octobre 2019, le taux a déjà atteint le niveau des années 2008, 2013, 2017.

\section{RÉSUMÉS}

La formule émergente politiquement incorrect gagne du terrain dans les discours publics qui circulent dans les sociétés démocratiques. Sur la base d'un corpus de discours médiatiques, numériques et savants des pays francophones et anglophones, l'article montre le rôle de plus en plus important du politiquement incorrect dans la vie sociale, et ses enjeux argumentatifs dans la sphère politique.

L'analyse de la définition discursive a permis de cerner les tendances à un usage croissant de la formule, ses dimensions - sur le plan du verbe et de l'action -, ainsi que les valeurs-clés qui y sont attachées : la jouissance d'une liberté absolue de pensée et, conséquemment, d'expression, contrairement aux limitations imposées par le politiquement correct; l'audace de prendre des risques pour énoncer une vérité parfois peu attrayante sans l'atténuer, comme l'exige le politiquement correct suivant le corpus scientifique. Si la définition discursive du politiquement incorrect a ses spécificités dans des milieux culturels différents, la formule est néanmoins définitivement entrée dans l'usage commun à partir des années 2000 et s'y est lexicalisée en produisant de nombreux dérivés et usages nuancés et périphrastiques.

Politically incorrect is an emergent formula that is gaining ground in the public discourses of democratic societies. On the basis of a corpus of media, digital and scholar discourses in French and English speaking countries, the article shows the increasingly important role of the politically incorrect discourse in social life and as well as its argumentative issues in the political sphere.

The discursive definition analysis has identified the tendencies of the increasing use of the formula, its dimensions-verbal and action related-, as well as the key values that are attached to it: enjoyment of an absolute freedom of thought and, consequently, of expression, contrary to the limitations imposed by the politically correct; the audacity to take the risk of stating a sometimes unattractive truth without mitigating it, as politically correct demands according to the scientific sources. The discursive definition of the politically incorrect has its specificities in the different cultural circles, nevertheless the formula has definitively entered into the common use since the 2000s and has lexicalized there as it shows numerous derivatives, nuanced and periphrastic uses. 
INDEX

Mots-clés : politiquement incorrect, incorrection politique, définition discursive

Keywords : politically incorrect, political incorrectness, discursive definition

\section{AUTEUR}

\section{MARIA SALTYKOV}

Doctorante, Université de Tel-Aviv, ADARR.

Maria Saltykov prépare actuellement une thèse sur le discours politique politiquement incorrect dans le cadre des stratégies électorales récentes en France (2017) et aux États-Unis (2016).

m.saltykova@gmail.com 